\title{
Is Emotional Suppression Always Bad? A Matter of Flexibility and Gender Differences
}

\author{
Guyonne Rogier $^{1} \cdot$ Carlo Garofalo $^{2} \cdot$ Patrizia Velotti $^{3}$
}

(C) Springer Science+Business Media New York 2017

\begin{abstract}
Although emotional suppression has usually been considered to be associated to psychopathological symptoms and aggression, different studies yielded controversial findings and highlighted possible gender differences in these relationships. In an attempt to cast light on this issue, we administered to a sample of 380 community-dwelling individuals the Emotion Regulation Questionnaire, the Aggression Questionnaire and the Symptom CheckList-90Revised. Gender differences (favoring women) emerged on the habitual use of cognitive reappraisal and expressive suppression. Interestingly, associations of emotional suppression were only significant among females, and mainly regarded psychological distress. In an attempt to test whether the flexibility in the use of emotion regulation strategies was more important for psychological well-being, we tested interaction effects between reappraisal and suppression on psychopathological diseases and aggression measures. Significant interaction effects were found among men and only on aggressive measures. Such results confirmed gender differences in emotion regulation and the relevance of flexibility in the use of emotional regulation strategies as part of adaptive emotional functioning.
\end{abstract}

Patrizia Velotti

patrizia.velotti@unige.it

1 Department of Dynamic and Clinical Psychology, Sapienza University of Rome, Rome, Italy

2 Department of Developmental Psychology, Tilburg University, Tilburg, The Netherlands

3 Department of Educational Sciences, University of Genoa, Genoa, Corso Andrea Podestà 2, 16128 Genoa, Italy
Keywords Emotion regulation - Aggression · Psychopathology $\cdot$ Gender differences · Emotional suppression $\cdot$ Cognitive reappraisal

The concept of emotion regulation refers to a set of processes that people use to modulate, consciously or not, their emotional states in order to respond to environmental requests in an adaptive way (Gross 1998). The Process Model of Emotion Regulation (Gross 1998) distinguishes between antecedentfocused strategies, which precede the emotional response, and response-focused strategies, which are initiated when the emotion is already under way. Cognitive reappraisal, an example of the former category, describes the process of connecting a particular meaning to a situation in order to regulate the imminent emotional experience. In contrast, emotional suppression is a response-focused strategy that refers to deliberate attempts to limit one's emotionally expressive behavior when aroused. Research in this area has led to a functional differentiation of these strategies based on their ability to facilitate adaptive (as opposed to a maladaptive) responses. For instance, it has been demonstrated that the use of adaptive emotion regulation strategies such as reappraisal was correlated with indicators of good psychological functioning while the use of maladaptive strategies, including suppression, is generally associated with overall psychopatology (Gross and John 2003).

An emotion regulation perspective has been widely adopted in the study of internalizing disorders such as depression or anxiety disorders. Indeed, an inability to cope with negative emotions is considered a central feature of depression and anxiety (Aldao et al. 2010; Gross and Muñoz 1995). A difficulty in regulating emotions has also been linked with psychosomatic symptoms (Lundh et al. 2001). In particular, psychosomatic disease has been linked to alexithymic features 
(i.e., difficulties in identifying, labeling, and describing one's feelings) as well as to a general deficit in the ability to tolerate negative emotions and to connect them to physical sensations (Subic-Wrana et al. 2010). Finally, recent findings suggest that emotion dysregulation can also characterize psychotic disorders (Garofalo and Velotti 2015).

In recent years, research on emotion regulation has also been conducted in the study of externalizing behavior and psychopathology (Aldao et al. 2010). Specifically, an emotion regulation framework has also been applied to the study of aggression and violent behavior, especially among male offenders (Roberton et al. 2014). For instance, emotional suppression in response to negative mood induction has been shown to predict displaced aggression using an experimental procedure among undergraduate students (Scott et al. 2015). Moreover, it has recently been reported that a more general deficit in emotion regulation could characterize violent offenders, and that overall emotion dysregulation was related to hostility, anger, trait aggression, and actual aggressive behavior (Garofalo et al. 2016; Roberton et al. 2014).

In an effort to advance research on the association between emotion regulation strategies and maladaptive outcomes, several authors proposed that, rather than being inherently adaptive or maladaptive, each emotion regulation strategy could be either adaptive or maladaptive in different contexts or depending on different situational contingencies (Webb et al. 2012). Accordingly, it has been proposed the importance of considering the flexibility in the use of emotion regulation strategies as an integral component of healthy functioning (Bonanno et al. 2004). In other words, it could be an impairment in an individual's ability to choose from different emotion regulation strategies and use them flexibly to be associated with maladaptive outcomes, rather than the use of an emotion regulation strategies per se (e.g., emotional suppression; Webb et al. 2012). For instance, Aldao and Nolen-Hoeksema (2011) reported that, in a sample of community residents, the negative association between adaptive emotion regulation strategies and psychopathological symptoms was moderated by levels of maladaptive strategies. Likewise, recent evidence suggests that emotional suppression is not always and necessarily linked to negative outcomes in nonclinical samples (e.g., Bonanno et al. 2004) and specifically some studies did not find a relation between suppression and depressive symptoms (Flynn et al. 2010). Similarly, the positive associations between cognitive reappraisal and individual well-being have been inconsistent across studies (e.g., Webb et al. 2012).

Finally, there is substantial evidence that the use of emotion regulation strategies may vary across gender. Indeed, women usually report a greater use of reappraisal than men (NolenHoeksema 2012), whereas men use suppression more often than women (Flynn et al. 2010). It has been argued that women are particularly prone to use reappraisal because they would appraise events as more stressful (Tamres et al. 2002) and report greater affect intensity comparing to men (Fujita et al. 1991). In a similar way, women could have been educated to pay deliberate attention to their emotions while male gender socialization would lead men to inhibit emotional expression (Gross and John 1998). Further, gender differences could also have an impact on the relationships between emotion dysregulation and both internalizing and externalizing symptoms (Nolen-Hoeksema 2012). Actually, different patterns have been reported when investigating the association between maladaptive emotion regulation and both depressive (Flynn et al. 2010) and anxious symptoms (Bardeen and Stevens 2015), comparing men and women. Flynn et al. (2010) found that the effect of emotional suppression on depressive symptomatology was more pronounced among men in a sample of undergraduate students. On the other hand, emotional inhibition was associated with anxiety only among women (Bardeen and Stevens 2015). Similarly, gender differences have also been found in the relation between emotion dysregulation and aggression (e.g., Bowie 2010). For instance, sex moderated the mediating role of emotion dysregulation in the association between negative affectivity and physical aggression, such that emotion dysregulation was more strongly related to aggression among men in a nonclinical sample (Donahue et al. 2014). Likewise, in a sample of students from undergraduate psychology courses, emotion dysregulation has been found to mediate the association between childhood maltreatment and intimate partner abuse only among men (Gratz et al. 2009). In contrast, Bowie (2010) found that poor emotion regulation predicted aggression among women only. However, Bowie examined this relationship in a sample of children and young adolescents, given a possible explanation for such contrasting results.

In light of the above conceptual and empirical work, in the present study we sought to examine, in a large community sample, gender differences in the use of two widely studied emotion regulation strategies (i.e., suppression and reappraisal) as well as in their associations with internalizing (i.e., psychopathological symptoms) and externalizing (i.e., aggression dimensions) spectra. It has been pointed out the importance to consider flexibility in emotion regulation but, to our knowledge, studies did not examine if the capacity to resort in a flexible way to emotion regulation strategies is differentially associated with negative aspects of psychological fuctionning across gender. Moreover, no previous study examined such topic in internalizing and externalizing spectra simultaneously. In line with previous studies (e.g., Flynn et al. 2010; NolenHoeksema 2012), we expected that on average women would use reappraisal more frequently than men, which in turn were expected to show greater levels of suppression. As for the other constructs, we expected men to report higher levels of physical aggression, whereas women were expected to report greater levels of psychopathological distress, especially depressive and anxiety symptoms. However, given the 
contrasting findings reported to date examining both the internalizing (e.g., Bardeen and Stevens 2015; Flynn et al. 2010; Nolen-Hoeksema 2012) and externalizing (e.g., Bowie 2010; Donahue et al. 2014; Gratz et al. 2009) spectra, no specific hypotheses could be drawn regarding gender consistency in the associations between emotion regulation strategies, psychopathological symptoms, and aggression. Tentative hypotheses based on recent findings were that the associations between reappraisal (negative) and suppression (positive) with psychopathological distress were stronger among women, whereas associations with aggression were stronger among men. Finally, in order to corroborate the relevance of flexible emotion regulation, we tested whether the interaction between the use of suppression and the use of reappraisal could further explain levels of psychopathological distress and aggression.

\section{Material and Methods}

\section{Participants and Procedure}

The sample consisted of 379 (66.1\% women, $N=251)$ community participants, who volunteered to participate in the study after providing written informed consent. Participants were recruited by means of a snowball sampling technique. Undergraduate students in psychology course were asked to complete the measures and to administer the battery of questionnaires to friends or family members. All participants resided in Genoa, Italy. No compensation was provided for participating at the study. Men $\left(M_{\text {age }}=31, S D=11.41\right)$ were significantly older than women $\left(M_{\text {age }}=28.50 ; S D=9.88\right)$, $t=2.11 ; p<.05)$. All procedures were approved by the Ethical Review Committee of the Department of Dynamic and Clinical Psychology at Sapienza University of Rome.

\section{Measures}

Aggression The Aggression Questionnaire developed by Buss and Perry in 1992 was used to assess trait aggression. The 29 items of this scale are rated on a 5-point Likert scale, and produce scores on four factors: Anger; Hostility; Physical aggression; and Verbal aggression. The validation study of the AQ demonstrated a good test-retest reliability over 9-weeks and a good internal consistency (Buss and Perry 1992). The Italian validation of the AQ (Fossati et al. 2003) confirmed its good psychometric properties in terms of factor structure and internal consistency In the present study, internal consistency coefficients for all scales ranged between .76 (Verbal Aggression) to .83 (Physical Aggression), with the total score showing a Cronbach's $\alpha$ of .90 .

Emotion Regulation The Emotion Regulation Questionnaire (ERQ) developed by Gross and John (2003) was used to assess Cognitive Reappraisal $(\alpha=.87)$ and Emotional Suppression $(\alpha=.79)$. The ERQ consists of 10 items rated on a 7-point Likert scale. Balzarotti et al. (2010) have demonstrated good internal consistency and 2-month test-retest reliability for the Italian version.

Psychological Distress The Symptom Checklist-90-Revised (SCL-90-R; Derogatis and Lazarus 1994) is a self-report checklist that measures the presence and the severity of psychological distress in the last month. The 90 items are rated on a 5-point Likert scale. This instrument encompasses nine symptom subscales: somatization ( $\alpha=.82)$, obsessioncompulsion $(\alpha=.84)$, interpersonal sensitivity $(\alpha=.84)$, depression $(\alpha=.90)$, anxiety $(\alpha=.84)$, hostility $(\alpha=.76)$, phobic anxiety $(\alpha=.75)$, paranoid ideation $(\alpha=.77)$, and psychoticism $(\alpha=.81)$. The SCL-90-R also provides a score indicating the overall level of psychological distress (Global Severity Index, GSI) which exhibited excellent internal consistency $(\alpha=.97)$ Therefore, the instrument has confirmed the good internal consistency of the original version $(\alpha=.70-.96)$ as well as the reliability and validity of the Italian SCL-90-R, as provided by Prunas et al. (2012).

\section{Data Analytic Approach}

Univariate (ANOVA) and multivariate (MANOVA) analyses of covariance (controlling for age) were carried out to compare men and women on all study variables, as appropriate. Because empirical evidences showed that age influences levels of emotion regulation (John and Gross 2004) and aggression (Fossati et al. 2003), we introduced this variable as a covariate. Partial Eta squared $\left(\eta_{p}^{2}\right)$ was used as an estimate of the effect size of the univariate $F$ tests. Partial correlations (controlling for age) between ERQ variables and AQ variables and between ERQ variables and SCL-90-R variables were calculated separately for men and women. To test the interaction effect between the use of reappraisal and the use of suppression on the levels of aggression and psychopathological distress, we split the sample by gender, centered variables on means for men and women separately and used Linear Regression Method entering age as a covariate. We used the PROCESS macro for SPSS 22.0 (Hayes 2013) in order to evaluate accurately statistical significances of the interaction effects at different levels of the independent variable.

\section{Results}

Table 1 shows means and standard deviations for aggression dimensions, emotion regulation strategies and psychological distress scales, as well as group comparisons across gender. In line with the expectations, men reported higher levels of 
Table 1 Means, standard deviations (SD) and group comparison (controlling for age) for all study variables $(N=379)$

\begin{tabular}{|c|c|c|c|c|c|c|c|c|}
\hline & \multicolumn{2}{|c|}{$\operatorname{Men}(N=128)$} & \multicolumn{2}{|c|}{ Women $(N=251)$} & \multicolumn{2}{|c|}{ Group Comparison } & \multicolumn{2}{|c|}{ Total $(N=379)$} \\
\hline & $M$ & $S D$ & $M$ & $S D$ & $F$ & $\eta_{p}^{2}$ & $M$ & $S D$ \\
\hline \multicolumn{9}{|l|}{ AQ } \\
\hline Physical Aggression & 17.48 & 6.48 & 14.13 & 5.34 & $29.26^{* * * *}$ & .07 & 15.26 & 5.95 \\
\hline Verbal Aggression & 14.35 & 3.74 & 13.66 & 4.24 & 2.59 & .00 & 13.89 & 4.09 \\
\hline Anger & 15.50 & 5.25 & 16.09 & 5.30 & 0.82 & .00 & 15.89 & 5.28 \\
\hline Hostility & 16.31 & 5.03 & 18.29 & 5.73 & $8.44^{* *}$ & .02 & 17.62 & 5.58 \\
\hline Total Score & 63.61 & 16.57 & 62.18 & 15.75 & $1.14^{* *}$ & .00 & 62.66 & 16.02 \\
\hline \multicolumn{9}{|l|}{ ERQ } \\
\hline Reappraisal & 4.72 & 1.02 & 4.97 & 1.05 & $4.93^{*}$ & .01 & 4.88 & 1.05 \\
\hline Suppression & 3.70 & 1.20 & 3.18 & 1.24 & $12.98^{* * *}$ & .03 & 3.35 & 1.24 \\
\hline \multicolumn{9}{|l|}{ SCL-90-R } \\
\hline Somatization & .55 & .42 & .83 & .60 & $22.25^{* *}$ & .06 & .74 & .56 \\
\hline Obsessive-compulsive & .80 & .57 & 1.00 & .71 & $6.96^{* *}$ & .02 & .94 & .67 \\
\hline Interpersonal sensitivity & .50 & .46 & .78 & .66 & $15.22^{* * *}$ & .04 & .69 & .61 \\
\hline Depression & .60 & .51 & .98 & .73 & $24.84^{* * *}$ & .06 & .85 & .69 \\
\hline Anxiety & .55 & .42 & .81 & .65 & $16.11^{* * * *}$ & .04 & .72 & .59 \\
\hline Hostility & .61 & .58 & .68 & .59 & 0.67 & .00 & .65 & .59 \\
\hline Phobic anxiety & .15 & .31 & .25 & .45 & $4.58^{*}$ & .01 & .22 & .41 \\
\hline Paranoid ideation & .66 & .58 & .84 & .69 & $5.43^{*}$ & .01 & .78 & .66 \\
\hline Psychoticism & .33 & .44 & .39 & .49 & 1.17 & .00 & .37 & .47 \\
\hline Global Severity Index & .54 & .38 & .76 & .53 & $15.55^{* * *}$ & .04 & .69 & .49 \\
\hline
\end{tabular}

$A Q$ Aggression Questionnaire, ERQ Emotion Regulation Questionnaire, SCL-90-R Symptom CheckList-90-Revised ${ }^{*} p<.05 ;{ }^{* *} p<.01 ;{ }^{* * *} p<.001$

physical aggression than women, which in turn showed higher levels of hostility. Furthermore, women reported to use more reappraisal and less suppression than men. Then, women scored higher than men on all subscales of the SCL-90-R except on the Psychoticism subscale, on which significant differences were not found.

As shown in Table 2, in line with our expectations, psychopathological symptoms were significantly associated with reappraisal (negatively) and suppression (positively), among women only. The only exception regarded Phobic anxiety, that was not associated with reappraisal. Among men, the only significant association regarded the negative association between reappraisal and somatization. Overall, these findings indicated that reappraisal was associated with psychological well-being, and suppression with psychological distress, among women only. Contrary to our expectations, associations between emotion regulation strategies and aggression dimensions were also significant only among women. Specifically, in the women sample, reappraisal was negatively associated with all aggression dimensions, whereas suppression was negatively related to verbal aggression and positively to hostility. Thus, higher levels of reappraisal were associated with fewer aggression tendencies only in women. Also, among women only, suppression was associated with less use of verbal aggression, but with greater hostility.

Finally, we examined if the interaction between levels of reappraisal and levels of suppression could bring further understanding of individuals differences in psychopathological symptoms or aggression. We tested the interaction hypotheses separately for men and women. Results are illustrated in Tables 3 and 4. We did not found any significant interaction among the women sample. Similarly, among men, levels of suppression and reappraisal did not seem to interact in the association with psychopathological distress. However, among men, we found a significant interaction effect between levels of emotion regulation strategies in relation to the total score of the AQ and the Hostility subscale $(p<.05$ for both dependent variables). Such results are illustrated in Figs. 1 and 2. Moreover, in the men group, the negative associations between reappraisal and AQ total score were significant only when suppression was high (for suppression $=+1 S D: b=-4.79, p<.05)$ while when suppression was low or moderate, reappraisal was not associated with AQ total score (for suppression $=-1 \mathrm{SD}: \mathrm{b}=1.32$, $p>.05$; for suppression $=$ Mean: $\mathrm{b}=-1.74, p>.05)$. Similarly, the negative association between reappraisal and Hostility score was significant only when suppression 
Table 2 Partial correlations (controlling for age) between emotion regulation strategies (reappraisal and suppression), psychopathological distress, and aggression dimensions across gender

\begin{tabular}{|c|c|c|c|c|}
\hline & \multicolumn{2}{|l|}{$\operatorname{Men}(N=128)$} & \multicolumn{2}{|l|}{ Women $(N=251)$} \\
\hline & ERQ Reappraisal & ERQ Suppression & ERQ Reappraisal & ERQ Suppression \\
\hline \multicolumn{5}{|l|}{ SCL-90-R } \\
\hline Somatization & $-.26^{* * *}$ & -.12 & $-.17^{* *}$ & $.22^{* * * *}$ \\
\hline Obsessive-Compulsive & -.03 & .08 & $-.18^{* *}$ & $.35^{* * * *}$ \\
\hline Interpersonal sensitivity & -.12 & .10 & $-.14^{*}$ & $.28^{* * * *}$ \\
\hline Depression & -.04 & .15 & $-.22^{* * *}$ & $.31^{* * * *}$ \\
\hline Anxiety & -.11 & -.07 & $-.23^{* * *}$ & $.25^{* * * *}$ \\
\hline Hostility & -.11 & -.08 & $-.27^{* * * *}$ & $.18^{* * *}$ \\
\hline Phobic anxiety & -.13 & .08 & -.08 & $.18^{* *}$ \\
\hline Paranoid Ideation & -.07 & -.03 & $-.20^{* *}$ & $.24^{* * * *}$ \\
\hline Psychoticism & -.15 & .01 & $-.19^{* * *}$ & $.27^{* * * *}$ \\
\hline GSI & -.14 & .03 & $-.23^{* * * *}$ & $.31^{* * * *}$ \\
\hline \multicolumn{5}{|l|}{ AQ } \\
\hline Physical aggression & -.16 & -.05 & $-.33^{* * * *}$ & .10 \\
\hline Verbal aggression & .07 & -.08 & $-.13^{*}$ & $-.14^{*}$ \\
\hline Anger & -.04 & -.08 & $-.28^{* * * *}$ & -.03 \\
\hline Hostility & -.06 & .04 & $-.28^{* * * *}$ & $.28^{* * * *}$ \\
\hline Total score & -.08 & -.02 & $-.34^{* * * *}$ & .08 \\
\hline
\end{tabular}

SCL-90-R Symptom Checklist 90 Revised, GSI Global Severity Index of the SCL-90-R, AQ Aggression Questionnaire, ERQ Emotion Regulation Questionnaire

${ }^{*} p<.05 ;{ }^{* *} p<.01 ;{ }^{* * *} p<.001$ was high (for suppression $=+1 S D: \mathrm{b}=-1.36, p<.05$ ) while reappraisal was not associated with hostility when suppression low (for suppression $=-1 \mathrm{SD}: \mathrm{b}=.45, p>.05$ ) or moderate for suppression $=$ Mean: $\mathrm{b}=-.46, p>.05)$. Then, results showed a significant negative interaction between suppression and reappraisal on the Anger subscale $(p<.05)$ as showed in Fig. 3. Finally, a significant interaction effect was also found for the Verbal Aggression subscale $(p<.05)$. Data showed that the positive association between reappraisal and verbal aggression was significant only at low levels of suppression (for suppression $=-1 S D: \mathrm{b}=.82, p<.05$ ) but reappraisal was not associated with Verbal Aggression when suppression was moderate (for suppression $=$ Mean: $\mathrm{b}=.13, p>.05$ ) or high (for suppression $=+1 \mathrm{SD}: \mathrm{b}=-.56, p>.05$ ).

\section{Discussion}

While connections among different forms of psychopathology and emotion regulation strategies are well-established, contrasting results are reported regarding the role played by gender in such associations. The present study contributes to the literature in this area in several ways. First, our results confirmed previous findings about gender differences in the use of emotion regulation strategies. Further, we added new evidence to support that associations between maladaptive emotion regulation (specifically, suppression), psychological distress, and aggression, might be more pronounced among women. Finally, the present research extends current knowledge about the role of flexibility in the use of emotion regulation for levels of well-being. Specifically, our results underlined how emotion regulations strategies are associated differently with aggression among men and women. Indeed, among men, it seems that aggression is not associated with the use of reappraisal or suppression per se (as seen among women) rather than with the capacity to use such strategies in a flexible way.

First, as extensively reported in the literature (e.g., NolenHoeksema 2012) women reported greater levels of psychopathological distress than men. Further, our findings regarding gender differences in aggression dimensions partially confirmed those of previous studies. As expected, men reported higher levels of physical aggression than women (Buss and Perry 1992). However, in our sample, no differences emerged on verbal aggression and anger, and women reported higher levels of hostility than men. This result contrasts with the study of Buss and Perry (1992), in which men were more hostile than women. However, it has been found elsewhere that Italian women seem to exhibit higher levels of hostility compared with other samples (Fossati et al. 2003). Our results can be interpreted in light of different socialization practices regarding masculinity and femininity. For example, it has been argued that gender socialization could lead men to avoid the expression of emotions, incoherent with the social male role, 


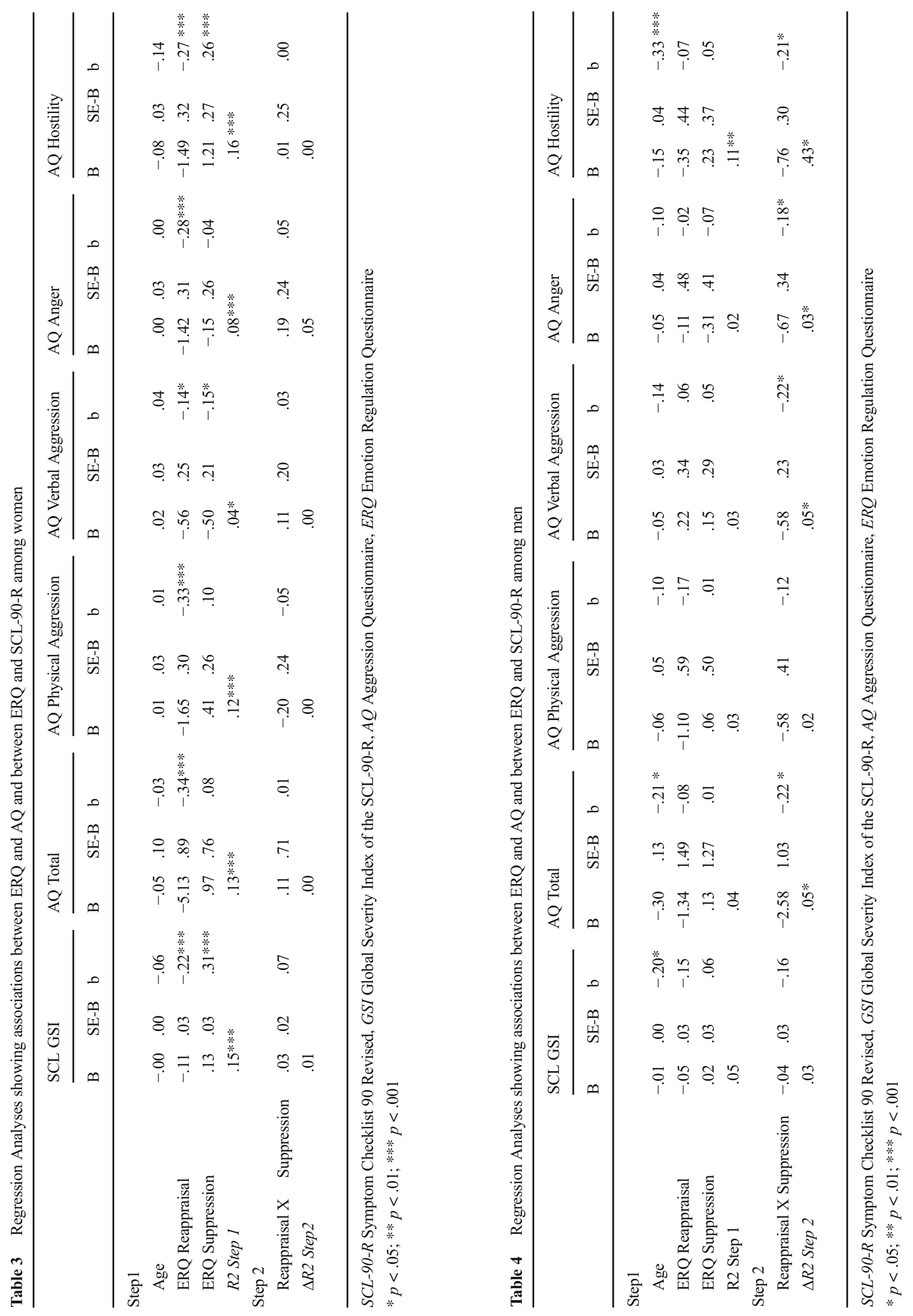


Fig. 1 Interaction Between Suppression and Reappraisal on AQ Total Scores among men

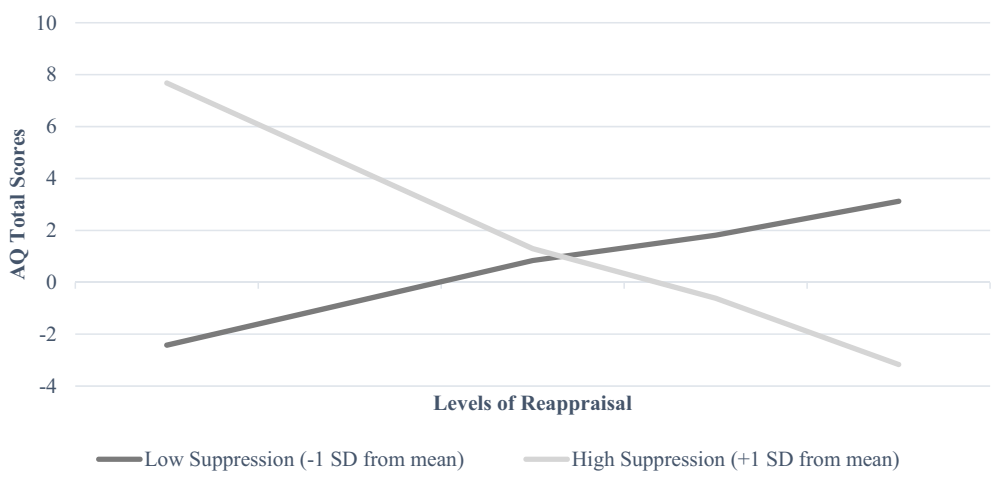

and to replace them with the expression of anger through violent and aggressive behavior (Gratz et al. 2009). Indeed, men showed an higher proneness to act in a physical aggressive way to externalize their aggressive tendencies, whereas it is possible that women resort to more indirect forms of (relational) aggression.

Results concerning gender differences in the use of emotion regulation strategies were largely consistent with previous studies (Flynn et al. 2010). Indeed, women reported to use more reappraisal than men, which in turn reported higher levels of suppression than women. In line with the gender socialization theory, it is possible to infer that men learn that they should conceal and deny their emotions throughout the development, or at least that suppressing emotions (rather than reappraising the eliciting situations) could be considered more appropriate in men than women. It is also possible that men are less in need of using of emotion and response focused strategies than women because more inclined to modulate the conditions that elicit emotions (trough selection of the situation or deployment of attention). In line with this possible explanation, and consistently with prior findings (Aldao et al. 2010) we found that, only among women, psychopathological distress was negatively related to reappraisal and positively related to suppression. These results might legitimate the functional differentiation between adaptive (emotion focused) and maladaptive (response focused) strategies.

Moreover, our results support the idea that the use of different emotion regulation strategies plays a key role in the development or in the maintenance of psychopathological symptoms in women, but not in men. It is possible that deviating from the use of emotion regulation strategies that are socially expected from women (i.e., high reappraisal, low suppression) is more detrimental than it is for men. Furthermore, it could be argued that psychopathological symptoms in men are linked to other maladaptive emotion regulation strategies, such as emotional avoidance, rumination or use of alcohol. The only exception regarded psychosomatic symptoms. Indeed, we found that also among men, reappraisal was negatively associated with psychosomatic symptoms. This result is consistent with prior studies that showed that psychosomatic disorders were associated with alexithymic traits (Subic-Wrana et al. 2010).

Examining correlations between each emotion regulation strategy and aggression dimensions, we found that the use of emotion regulation strategies, considering them separately, was associated with aggression among women only. This finding is consistent with the study by Bowie (2010), although other studies have reported the opposite pattern (e.g., Donahue et al. 2014; Gratz et al. 2009). It should be pointed out that our conclusions, drawn from a study on a sample of young adults, seem to confirmed evidences found among a sample of children and adolescents. Then, in our sample, women who made a greater use of reappraisal were less aggressive. We also found that women who were more hostile made a greater use of suppression and a lower use of reappraisal. Such results suggest that the use of reappraisal might be a relevant feature associated to aggressive tendencies among women, whereas an excessive use of suppression could account for the greater presence of hostility in women.

Interestingly, we found that women who are more verbally aggressive make a lower use of suppression. Such result
Fig. 2 Interaction Between Suppression and Reappraisal on Hostility among men

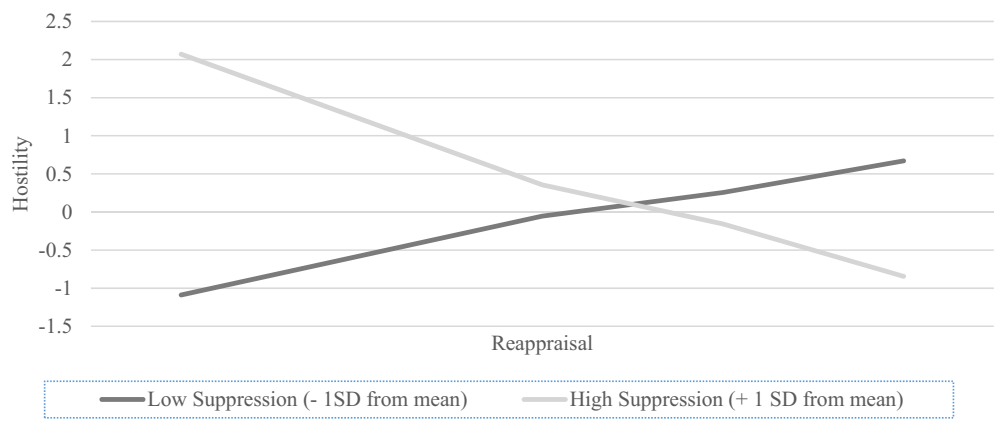


Fig. 3 Interaction Between Suppression and Reappraisal on Anger among men

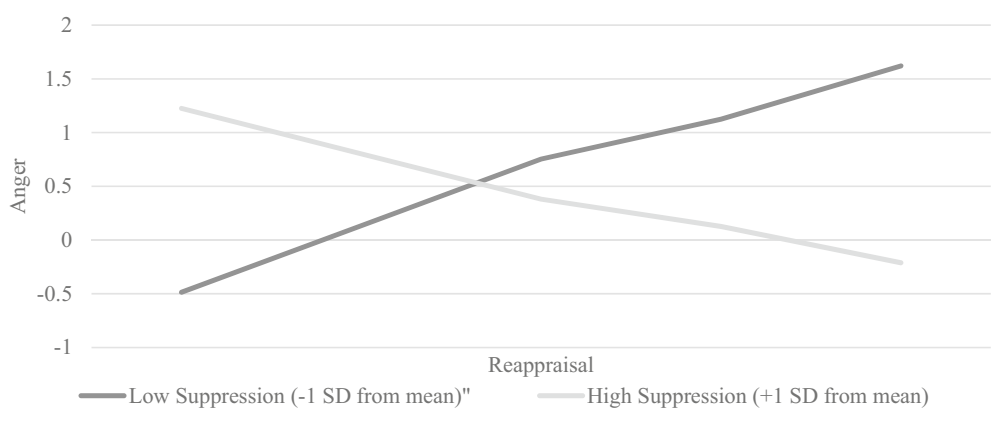

indicates a positive role played by suppression that could be explained considering the importance of context in evaluating the adaptive valence of regulation strategies. Indeed, it is easy to imagine how the suppression mechanism could be helpful in situation where it would not be appropriate express negative feelings openly. On the other hand, it should be noted that verbal aggression has been previously linked with measures of assertiveness (Buss and Perry 1992), and clearly represents a less maladaptive way to express aggressive tendencies than physical aggression. Therefore, another possible explanation of this association is that women who tend to suppress their feelings, do not express them. As such, it is plausible that when they experience angry, aggressive, or antagonistic feelings, they do not express them verbally, perhaps resorting to less adaptive strategies to cope with those negative emotions, such as physical aggression.

Looking at the interaction effects between suppression and reappraisal, interesting patterns emerged. Indeed, as discussed above, among women, suppression and reappraisal seem to play independent roles in externalizing psychopathology. In contrast, among men, we did not found significant correlations between the use of the emotion regulation strategies and aggression considering reappraisal and suppression as two independent strategies but analyses showed significant interactions between reappraisal and suppression, underlying a complex role played by emotional regulation in aggression among men. While it has been confirmed the absence of association between emotion regulation strategies and psychopathological symptoms among men, the examination of the interaction effects between these strategies evidenced a role played by emotional regulation in aggression. First, we found that reappraisal was positively associated with verbal aggression when men exhibited low levels of suppression. Such data constitutes an additional support to the conceptualization, discussed above, of verbal aggression as a more adaptive form of aggression. In that sense, reappraisal could operate as an adaptive strategy, allowing men to express negative feelings through verbal aggression rather than responding to such emotional states with more dysfunctional behaviors as physical aggression. Then, our results showed that reappraisal was negatively associated with aggression, hostility and anger only when men exhibited high levels of suppression. This result is line with prior findings suggesting that the negative association between adaptive emotion regulation strategies (here, reappraisal) and dysfunctional psychological functioning might actually depend on levels of maladaptive emotion regulation strategies (here, suppression; Aldao and NolenHoeksema 2011). Moreover, it seems that high levels of suppression are disadaptive only if men are not able to resort to other adaptive strategies. That is, the use of reappraisal per se could not be adaptive toward aggressive tendencies, rather this effect occurs only when suppression is strongly used. In that sense, we could think that reappraisal reveals itself as an adaptive regulation strategy only when men are using an excessive level of suppression. In a similar way, it is possible to think that high levels of suppression are disadaptive only when coupled with low levels of reappraisal. With a certain degree of inference, it is possible a specific pattern (high suppression, high reappraisal) describes a group of men who are able to flexibly select and use different emotion regulation strategies in different situations. We could think that the fact that gender socialization may lead to a greater use of suppression could not constitute a disadaptive situation per se, but the dysfunctional pattern may emerge when men are not able to be flexible in their use of emotion regulation strategy in resorting to alternative ones (as reappraisal). If women are educated to mainly resort to reappraisal, it is more evident why the use of suppression, emerging as a dysfunctional strategy also at low levels, could be primarily associate with low well-being. Men could have been educated to use suppression under certain social limits and the disadaptive aspect of such strategy could emerge only when others psychological resources are lacking. This would be consistent with the relevance of flexibility in the use of emotion regulation strategies as part of adaptive emotional functioning (Bonanno et al. 2004). Therefore, these findings seem to suggest that interventions aimed at reducing the aggression among men should primarily focus on diminishing the use of suppression while in women an intervention on both strategies seems recommended.

The present study has several limitations. First, we only used self-report measures, which might be affected by response bias, and might fail to capture aspects that are more aptly assessed via clinical interviews, informant-report, or laboratory tasks. For example, it could be argued that men tend to score lower than 
women on the self-report measures of emotion regulation strategies not because they do not use such strategies but because they lack insight into these mechanisms. The use of self-report only could have also inflated inter-correlations due to common method variance. Others limitations are related to the nature of our sample. The participation was voluntary and subjects were not representative of the general population. Further, our sample was neither a clinical nor a forensic population, therefore caution should be adopted when generalizing our results to more severely disturbed or violent populations. An important limitation of our study is that we measured only two strategies of emotion regulation (reappraisal and suppression). However, other emotion regulation strategies have recently been linked to psychological distress and aggression, such as emotional avoidance, rumination and drinking to cope. Finally, the cross sectional design of our study does not allow us to draw conclusions about the direction of the relations between constructs. It remains unknown if the use of reappraisal and suppression are mechanisms responsible for the outcomes (or for their maintenance), if psychological distress and aggression shape emotion regulation styles, or if a reciprocal influence takes place.

Keeping these limitations in mind, our study provides some support and additional information on the interrelations among emotional regulation strategies, psychopathological symptoms, and aggression across gender. Our findings confirmed previous studies about the role of emotion regulation strategies among women and suggest that the (un)balance in the use of cognitive reappraisal and emotional suppression might be more relevant for men's adaptive functioning. Specifically, comparing to women, reappraisal could be less adaptive in men as a strong use of this strategy could not associated with less aggression when the level of suppression remain moderated. On the whole, our study supports the importance of studying and treating emotion regulation placing emphasis on both maladaptive and adaptive strategies, as well as on the flexibility in their use.

\section{Compliance with Ethical Standards}

Ethical Approval All procedures performed in studies involving human participants were in accordance with the ethical standards of the institutional and/or national research committee and with the 1964 Helsinki declaration and its later amendments or comparable ethical standards.

Informed Consent Informed consent was obtained from all individual participants included in the study.

\section{References}

Aldao, A., \& Nolen-Hoeksema, S. (2011). When are adaptive strategies most predictive of psychopathology? Journal of Abnormal Psychology, 121(1), 276-281.
Aldao, A., Nolen-Hoeksema, S., \& Schweizer, S. (2010). Emotionregulation strategies across psychopathology: a meta-analytic review. Clinical Psychology Review, 30(2), 217-237.

Balzarotti, S., John, O. P., \& Gross, J. J. (2010). An Italian adaptation of the emotion regulation Questionnaire. European Journal of Psychological Assessment, 26(1), 61-67.

Bardeen, J. R., \& Stevens, E. N. (2015). Sex differences in the indirect effects of cognitive processes on anxiety through emotion regulation difficulties. Personality and Individual Differences, 81, 180-187.

Bonanno, G. A., Papa, A., Lalande, K., Westphal, M., \& Coifman, K. (2004). The importance of being flexible: the ability to both enhance and suppress emotional expression predicts long-term adjustment. Psychological Science, 15(7), 482-487.

Bowie, B. H. (2010). Understanding the gender differences in pathways to social deviancy: relational aggression and emotion regulation. Archives of Psychiatric Nursing, 24(1), 27-37.

Buss, A. H., \& Perry, M. (1992). The aggression Questionnaire. Journal of Personality and Social Psychology, 63(3), 452-459.

Derogatis, L. R., \& Lazarus, L. (1994). SCL-90-R, brief symptom inventory, and matching clinical rating scales. In M. E. Maruish \& M. E. Maruish (Eds.), The use of psychological testing for treatment planning and outcome assessment (pp. 217-248). Hillsdale: Lawrence Erlbaum Associates, Inc..

Donahue, J. J., Goranson, A. C., McClure, K. S., \& Van Male, L. M. (2014). Emotion dysregulation, negative affect, and aggression: a moderated, multiple mediator analysis. Personality and Individual Differences, 70, 23-28.

Flynn, J. J., Hollenstein, T., \& Mackey, A. (2010). The effect of suppressing and not accepting emotions on depressive symptoms: is suppression different for men and women? Personality and Individual Differences, 49(6), 582-586.

Fossati, A., Maffei, C., Acquarini, E., \& Di Ceglie, A. (2003). Multigroup confirmatory component and factor analyses of the Italian version of the aggression Questionnaire. European Journal of Psychological Assessment, 19(1), 54-65.

Fujita, R., Diener, E., \& Sandvik, E. (1991). Gender differences in negative affect and well-being: the case for emotional intensity. Journal of Personality and Social Psychology, 41, 427-434.

Garofalo, C., \& Velotti, P. (2015). Alcohol misuse in psychiatric patients and nonclinical individuals: the role of emotion dysregulation and impulsivity. Addiction Research and Theory, 23(4), 294-300.

Garofalo, C., Holden, C. J., Zeigler-Hill, V., \& Velotti, P. (2016). Understanding the connection between self-esteem and aggression: the mediating role of emotion dysregulation. Aggressive Behavior, 42, 3-15.

Gratz, K. L., Paulson, A., Jakupcak, M., \& Tull, M. T. (2009). Exploring the relationship between childhood maltreatment and intimate partner abuse: gender differences in the mediating role of emotion dysregulation. Violence and Victims, 24(1), 68-82.

Gross, J. J. (1998). The emerging field of emotion regulation: an integrative review. Review of General Psychology, 2(3), 271-299.

Gross, J. J., \& John, O. P. (1998). Mapping the domain of expressivity: multimethod evidence for a hierarchical model. Journal of Personality and Social Psychology, 74, 170-191.

Gross, J. J., \& John, O. P. (2003). Individual differences in two emotion regulation processes: implications for affect, relationships, and wellbeing. Journal of Personality and Social Psychology, 85(2), 348362.

Gross, J. J., \& Muñoz, R. F. (1995). Emotion regulation and mental health. Clinical Psychology: Science and Practice, 2(2), 151-164.

Hayes, A. F. (2013). Introduction to mediation, moderation, and conditional process analysis: a regression-based approach. New York: Guilford Publications Inc..

John, O. P., \& Gross, J. J. (2004). Healthy and unhealthy emotion regulation: personality processes, individual differences, and life span development. Journal of Personality, 72, 1301-1333. 
Lundh, L.-G., Wikström, J., \& Westerlund, J. (2001). Cognitive bias, emotion, and somatic complaints in a normal sample. Cognition and Emotion, 15(3), 249-277.

Nolen-Hoeksema, S. (2012). Emotion regulation and psychopathology: the role of gender. Annual Review of Clinical Psychology, 8, 161-187.

Prunas, A., Sarno, I., Preti, E., Madeddu, F., \& Perugini, M. (2012). Psychometric properties of the Italian version of the SCL-90-R: a study on a large community sample. European Psychiatry, 27(8), 591-597.

Roberton, T., Daffern, M., \& Bucks, R. S. (2014). Maladaptive emotion regulation and aggression in adult offenders. Psychology, Crime \& Law, 20(10), 933-954.

Scott, J. P., DiLillo, D., Maldonado, R. C., \& Watkins, L. E. (2015). Negative urgency and emotion regulation strategy use: associations with displaced aggression. Aggressive Behavior, 41(5), 502-512.

Subic-Wrana, C., Beutel, M. E., Knebel, A., \& Lane, R. D. (2010). Theory of mind and emotional awareness deficits in patients with somatoform disorders. Psychosomatic Medicine, 72(4), 404-411.

Tamres, L. K., Janicki, D., \& Helgeson, V. S. (2002). Sex differences in coping behavior: a meta-analytic review and examination of relative coping. Personality and Social Psychology Review, 6, 2-30.

Webb, T. L., Miles, E., \& Sheeran, P. (2012). Dealing with feeling: a meta-analysis of the effectiveness of strategies derived from the process model of emotion regulation. Psychological Bulletin, 138(4), 775-808. 